\title{
Research Paper \\ Comparing the Electromyography Activity of Core Muscles During Side Plank Exercise on Stable and Unstable Surfaces
}

\author{
*Farideh Babakhani ${ }^{1}$ (D), Mohamadreza Hatefi² (D) \\ 1. Department of Corrective Exercises and Sport Injury, Faculty of Physical Education, Allameh Tabataba'i University, Tehran, Iran. \\ 2. Department of Corrective Exercises and Sport Injury, Faculty of Physical Education, Kharazmi University, Tehran, Iran.
}

$\begin{gathered}\text { Use your device to scan } \\ \text { and read the article online }\end{gathered}$
Cittation: Babakhani F, Hatefi M. [Comparing the Electromyography Activity of Core Muscles During Side Plank Exercise
on Stable and Unstable Surfaces (Persian)]. Journal of Sport Biomechanics. 2019; 5(2):102-111. https://doi.org/10.32598/biome-
chanics.5.2.3

\section{(i) (8)}

Article Info:

Received: 10 Feb 2019

Accepted: 05 Jul 2019

Available Online: $01 \mathrm{Sep} 2019$

Key words:

Core stability, Plank,

Surface electromyography

\begin{abstract}
Objective Numerous abdominal exercises with Swiss ball are used to improve core stability with strengthening and rehabilitation goals. It is claimed that the stability exercises have a greater impact on core muscle activation, but the validity of this claim is still in doubt. Moreover, there is no comprehensive study on the comparison of the core muscles activity in different core stability exercises. Therefore, the purpose of this study was to compare the Electromyography (EMG) activity of core muscles while performing side plank on stable and unstable (Swiss ball) surfaces.

Methods Fifteen male students of Allameh Tabataba'i University were selected for the study based on inclusion and exclusion criteria. The EMG activity of gluteus medius, rectus abdominis, external oblique, and internal oblique muscles in subjects was recorded while performing side plank exercise on a fixed surface and a Swiss ball ( 2 sets of 5 seconds with a 30 -second rest interval).

Results There was a significant difference in the EMG activity of gluteus medius, external oblique and rectus abdominis muscles between two conditions of with and without Swiss ball $(P<0.05)$, but no significant difference was observed in the EMG activity of internal oblique muscle ( $P>0.05)$.

Conclusion Side plank exercise on the unstable surfaces (Swiss balls) can cause changes in the EMG activity of gluteus medius, rectus abdominis and external oblique muscles compared to when the exercise is performed on stable surfaces, and highly involved the pelvic lumbar muscles effective in maintaining core stability. Therefore, the use of side plank exercise on unstable conditions in a progressive program with gradually increased intensity is recommended which can be effective in strengthening and applying effective muscle contractions useful for core stability.
\end{abstract}

\section{Extended Abstract}

\section{Introduction}

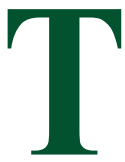

runk stability ensures the maintenance of the spinal alignment and proper control of movements in functional activities, and is vital for the prevention and treatment of lumbar injuries, including low back pain.
In this regard, several abdominal exercises with Swiss balls are used to improve core stability with strengthening and rehabilitation goals. It is claimed that stability exercises have a greater effect on activating the core muscles, but the correctness of this claim is still unclear, and few studies have compared the electrical activity of core muscles under different core stability exercises. Hence, this study aimed to compare the elec-

* Corresponding Author:

Farideh Babakhani, PhD.

Address: Department of Corrective Exercises and Sport Injury, Faculty of Physical Education, Allameh Tabataba'i University, Tehran, Iran.

Tel: +98 (918) 8318497

E-mail: farideh_babakhani@yahoo.com 
Table 1. EMG activity results of selected muscles under conditions of with and without Swiss ball

\begin{tabular}{|c|c|c|c|c|c|}
\hline \multirow{2}{*}{ Variables } & \multirow{2}{*}{ df } & \multicolumn{2}{|c|}{ Mean $\pm S D$} & \multirow{2}{*}{$\mathbf{t}$} & \multirow{2}{*}{$\mathbf{P}$} \\
\hline & & Without Swiss Ball & With Swiss Ball & & \\
\hline Rectus abdominis & 14 & $0.50 \pm 0.19$ & $0.55 \pm 0.16$ & 803.3- & $0.005^{*}$ \\
\hline Internal oblique & 14 & $0.0 \pm 23.08$ & $0.24 \pm 0.06$ & -1.749 & 0.102 \\
\hline External oblique & 14 & $0.56 \pm 0.11$ & $0.63 \pm 0.14$ & -3.770 & $0.002^{*}$ \\
\hline Gluteus medius & 14 & $0.21 \pm 0.16$ & $0.27 \pm 0.17$ & -6.167 & $0.001 *$ \\
\hline
\end{tabular}

tromyography (EMG) activity of the core muscles under side plank exercise on stable and unstable surfaces

\section{Participants and Methods}

In the present study, according to the entry and exit criteria and using a convenience sampling method, 15 male students of Allameh Tabataba'i University in Tehran, Iran were selected for the study. The EMG activity, using a 16-channel surface EMG system (Bayamed Co., Iran) of gluteus medius, rectus abdominis, external oblique, and internal oblique muscles of all subjects was recorded while performing side plank exercise ( 2 sets of 5 seconds with a 30 -second rest interval) on stable and unstable (Swiss ball) surfaces. Data from recording EMG activity of muscle with $1000 \mathrm{~Hz}$ sampling frequency and a bandwidth of 10-450 Hz filter (band-pass filtered), and the studied muscles were calculated and presented as Root Mean Score (RMS) and percentage of maximal voluntary isometric contraction (\% MVIC) and then were normalized. LabVIEW software (National Instruments, Austin, TX) was used to analyze raw signals. The raw were summarized in Excel and analyzed in SPSS v.21. To test the normality of data distribution, the Shapiro-Wilk test, was used, and paired t-test was used to compare the results between the two different plank conditions. The significance level was set at $\mathrm{P}<0.05$.

\section{Results}

The results of the statistical test (Table 1) showed a significant difference in the EMG activity of gluteus medius, external oblique and rectus abdominis muscles between conditions of with and without Swiss ball $(\mathrm{P}<0.05)$, but no significant difference was observed in the EMG activity of the internal oblique muscle $(\mathrm{P}>0.05)$.

\section{Conclusion}

Side plank exercise on unstable surfaces (Swiss ball) cause significant changes in EMG activity of gluteus medius, external oblique and rectus abdominis muscles compared to when the exercise is performed on a stable surface, and highly involved pelvic lumbar muscles affecting core stability. Therefore, the use of side plank exercise on unstable surfaces in a progressive program with gradually increased intensity can be effective in strengthening and using muscle contractions useful for core stability.

\section{Ethical Considerations}

\section{Compliance with ethical guidelines}

All subjects participated in the present study voluntarily after signing a consent form.

\section{Funding}

This study was extracted from a research proposal approved by Allameh Tabataba'i University.

\section{Authors' contributions}

All authors contributed equally in preparing this article.

\section{Conflicts of interest}

The authors declared no conflict of interest. 
This Page Intentionally Left Blank 


\title{
مقايسه فعاليت الكترومايوكَرافى عضالت ناحيه مركزى در حركت يّانك از يهلو روى سطح ثابث و سويُيس باليت
}

\author{
"فريده باباخانى' (1)، محمدرضا هاتفى ') \\ 1. كروه آسيبشناسى ورزشى و حركات اصلاحى، دانشكده تربيتبدنى و علوم ورزشى، دانشكاه علامه طباطبايیى، تهران، ايران.

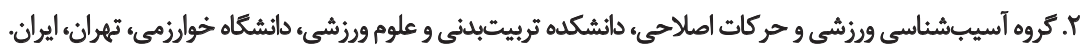

\begin{abstract}
حكدن

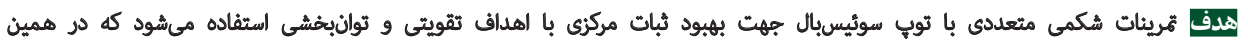

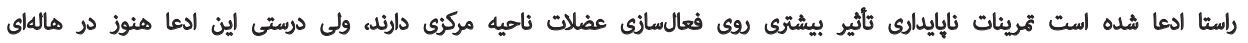

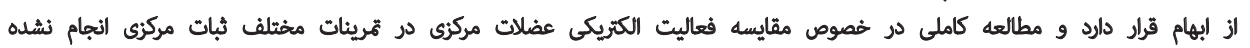

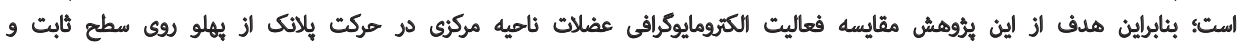
سوتيسبالبود.

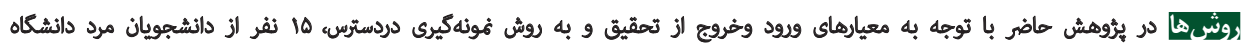

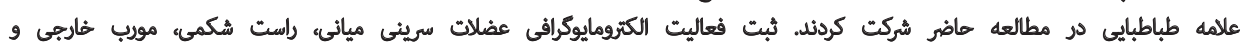

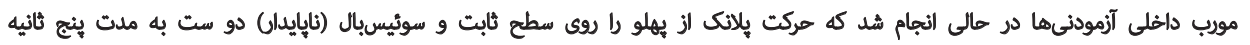

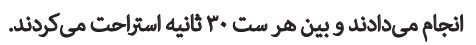

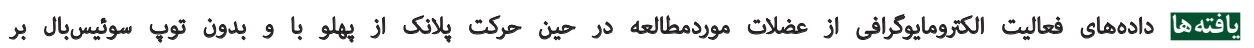

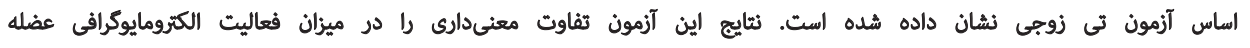

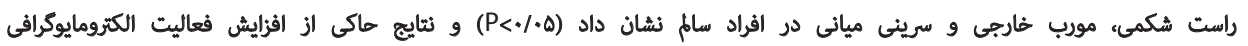

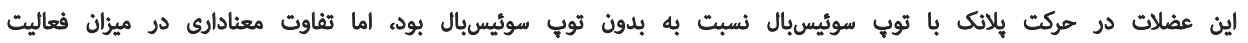

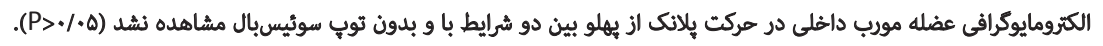

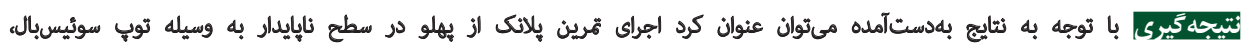

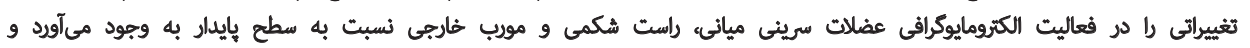

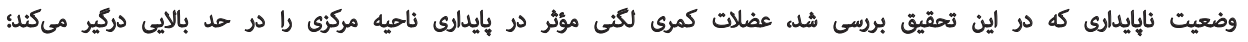

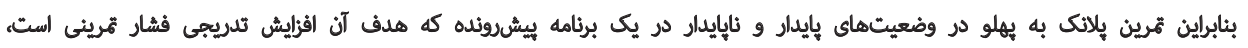

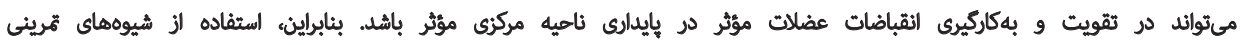

كمرى و از طريق كثترل سفتى بين مهرهاى به ثبات تنه كمك دئ

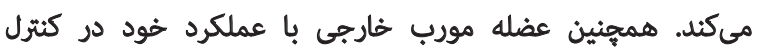

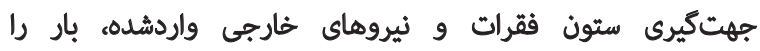

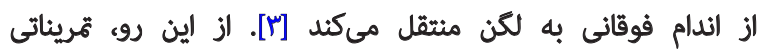

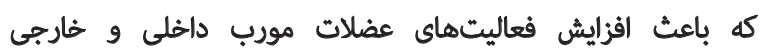

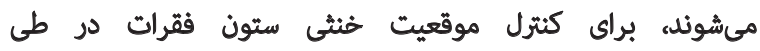

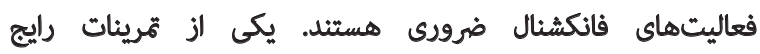
به منظور افزايش استقامت، قدرت و ثبات عضلات فيتات ناحيه مركز، حركت يلانك از يهلو است.

مقدمه ثبات ثنه، حفظ راسثاى ستون فقرات و كنترل مناسب حركات

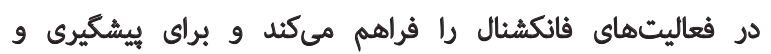

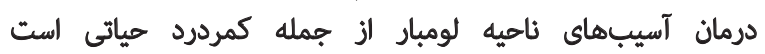

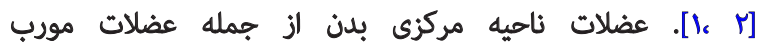

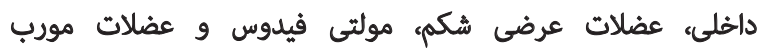

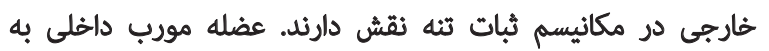

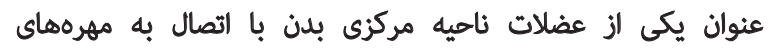


اين عضلات مانند تمرينات يل، كرانج و بهلانك و از طرفى خلأ

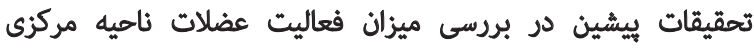

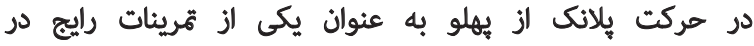

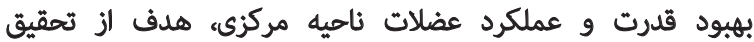

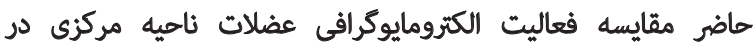

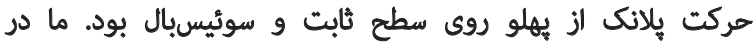

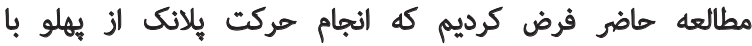
توب سوئيسبال باعث افزايش فعاليت الكترومايكرافى عضلات

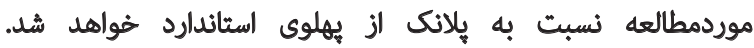

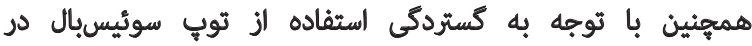
كلينيكهاى توانبخشى و ورزشى، اين سؤال مطرح شد كائ كه آيا

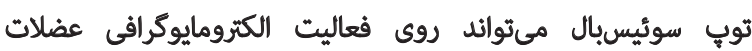
در حركت يلانك از يهلو تأثير بكذارد؟

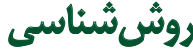

G*Power, Franz Faul Uni-) با توجه به نرم افزار جياوي و و با فرض هersity of Kiel, Germany

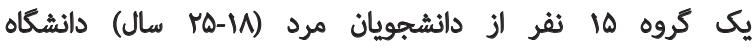

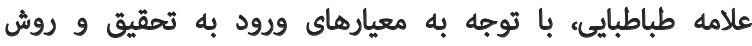

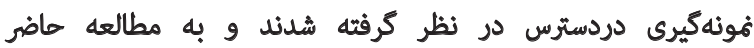

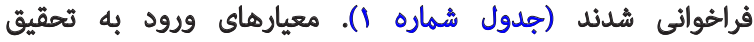

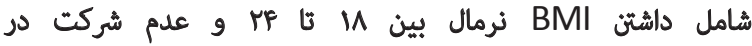

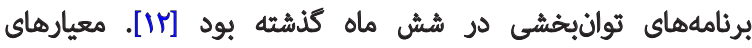

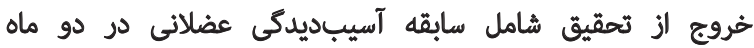

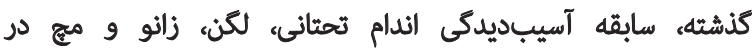

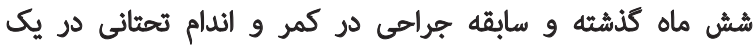

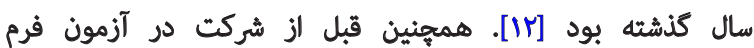

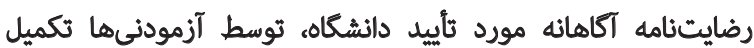

به طور كلى فعاليت الكترومايوكرافى (سيستم الكترومايوكرافى

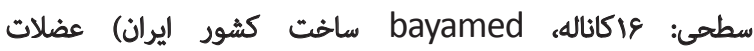
مورب خارجى، مورب داخلى، راست شكمى و سرينى ميانى همه إنها

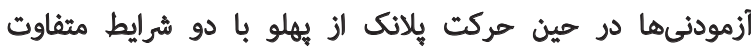

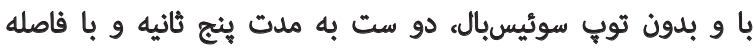

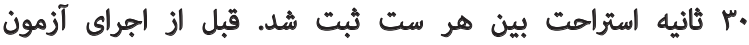

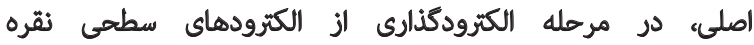

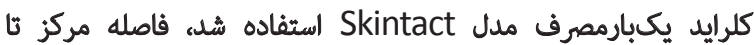

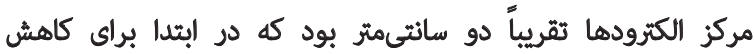

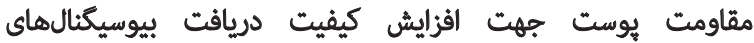

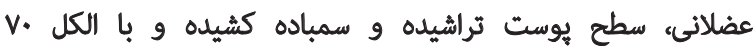

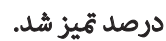

همجنين الكترودها در جهت فييرهاى عضلات بر اساس يروتكل SENIAM قرار كرفتند. محل نصب الكترود در عضله

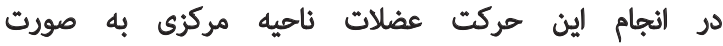

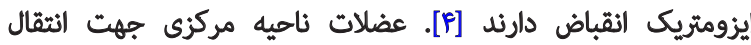

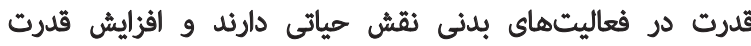

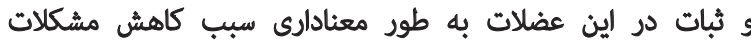

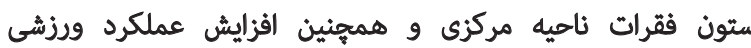

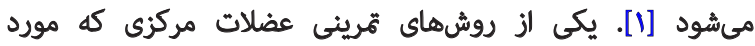

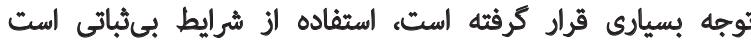

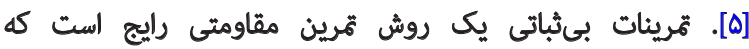

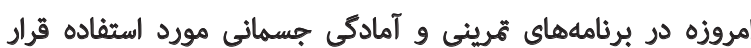

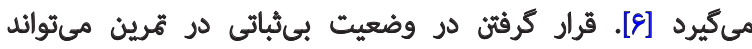

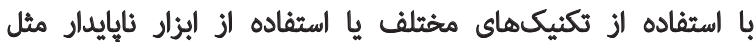

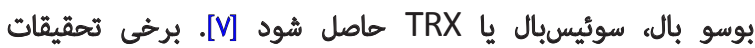

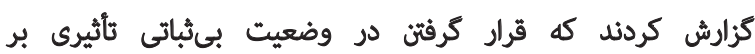

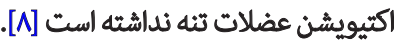

اما در همين راستا تمقيقات متعددى كزارش كردند كه تمرين

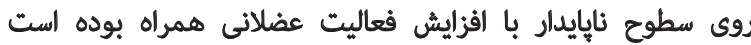

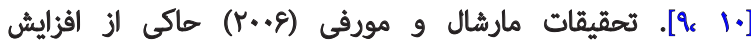

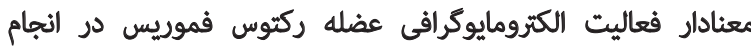

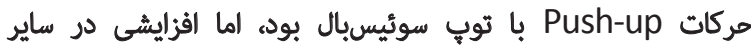

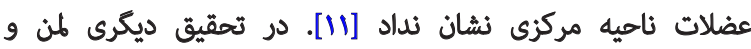

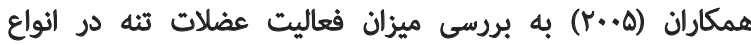

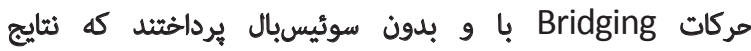

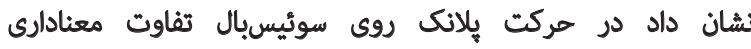

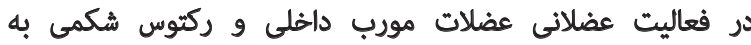

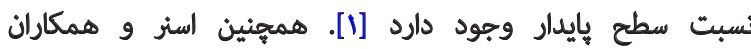

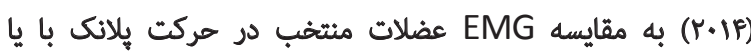

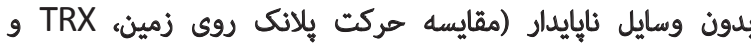

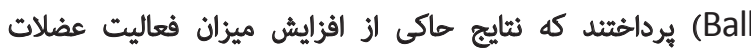

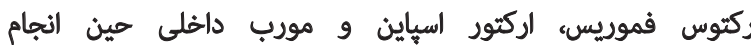

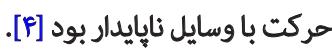

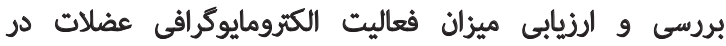

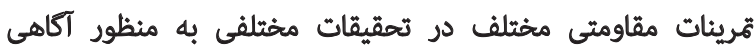

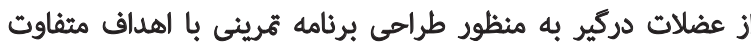

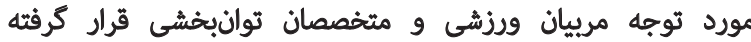

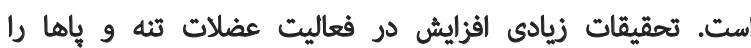

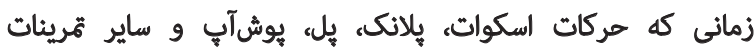

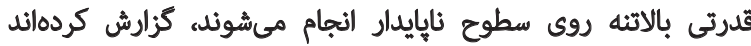

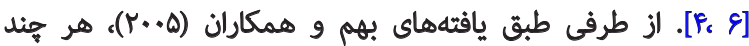

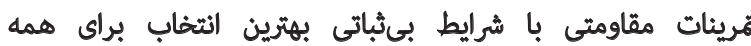

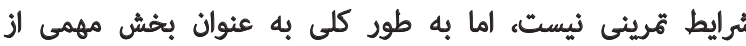

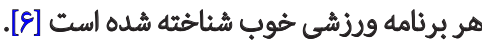
بنابراين با توجه به تثاقضات نتايج مطالعات بيشين در مورد تفاوت ميزان فعاليت عضلات ناحيه مركزى در تمرينات ويرهات نائه 
وجود داشت و ميانكين آن به عنوان MVIC در نظر كرفته شد

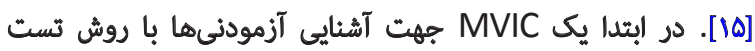

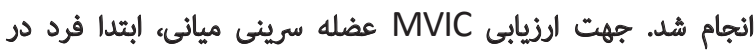

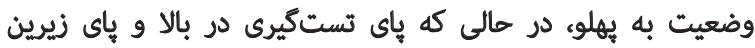

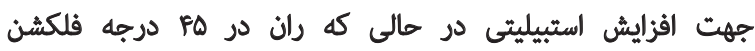

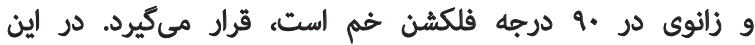

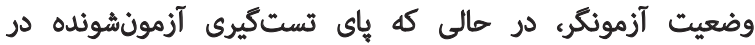

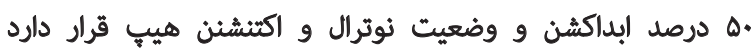

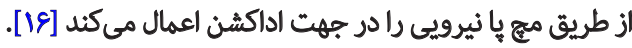

جهت ارزيابى MVIC عضله راست شكمى، ابتدا فرد در

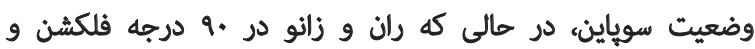

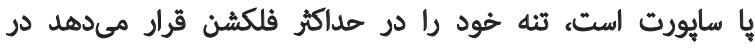

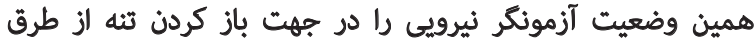

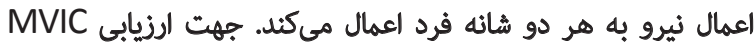

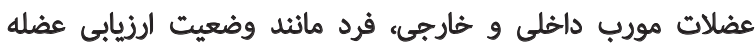

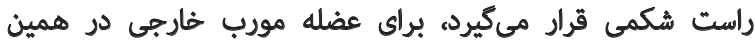

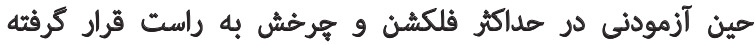

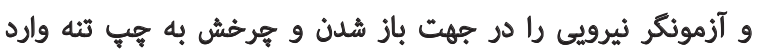

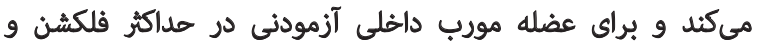

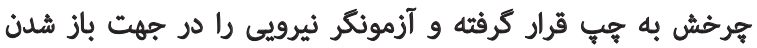

مورب خارجى در فاصله 10 سانتىمترى الز ناف و در نصف فاصله

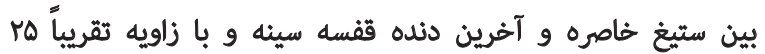

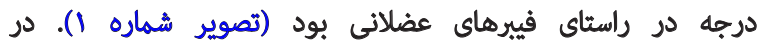
عضله مورب داخلى، در مركز هندسى مثلث فرضى تشكيل شئه فئه

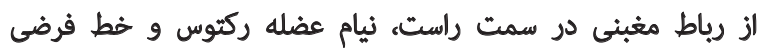
بين ناف و ASIS و در راست شكمى، تقريباً در دو سائتىمتر بيرون

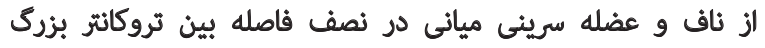

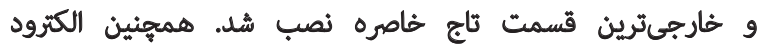

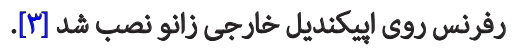

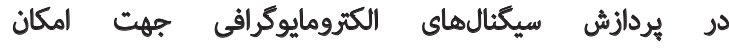

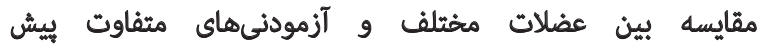

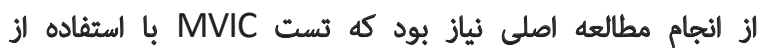

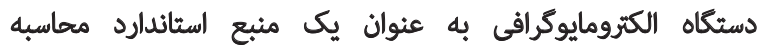

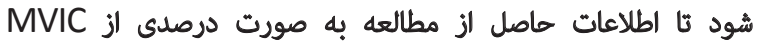

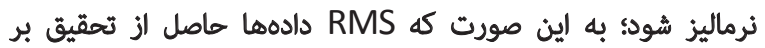
RMS

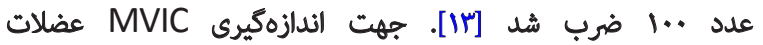

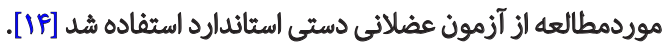
براى هر عضله، دو MVIC به مدت ينج ثائيه النجام شد كه جهت كاهش اثر خستكى بين هر تكرار يك دقية دونه استراحت

جدول ا. مشخصات جمعيتشئاحتي آزمودني هاي شركت كثئله در مطالعه (n=1ه)

\begin{tabular}{|c|c|c|}
\hline تى & ميانكين土|نحر أف معيار & مثتغير \\
\hline .118 & $M \in / N I \pm I / T a$ & سن (years) \\
\hline.$/ M f$ & $V \cdot / 9 T \pm T / F \Delta$ & جرم (Kg) \\
\hline. $\mid+1$ & IW/EIEY/AY & قد (cm) \\
\hline . $/ \Delta r$ & $M T / e f \pm=/ \Delta r$ & شاخص توده بلنى (BMI) \\
\hline
\end{tabular}

مجله بيومكانيك وزنث

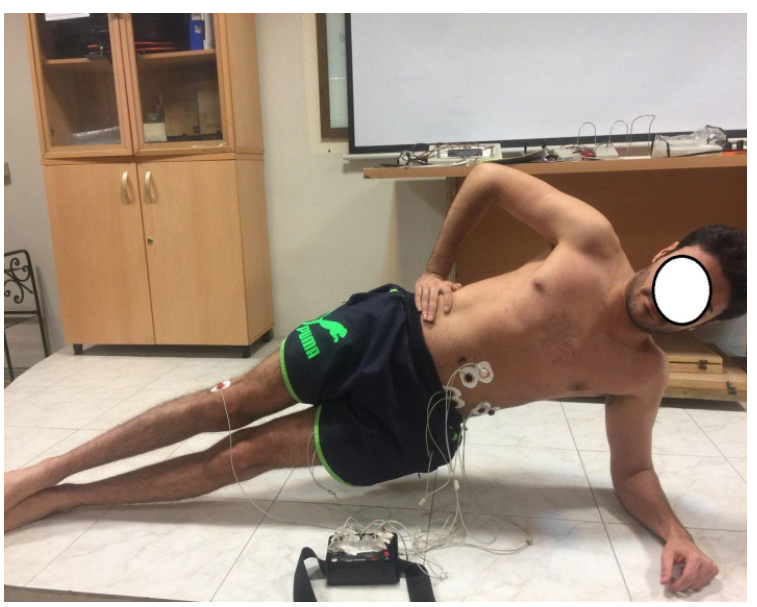

مجله بيومكانيك وزنش

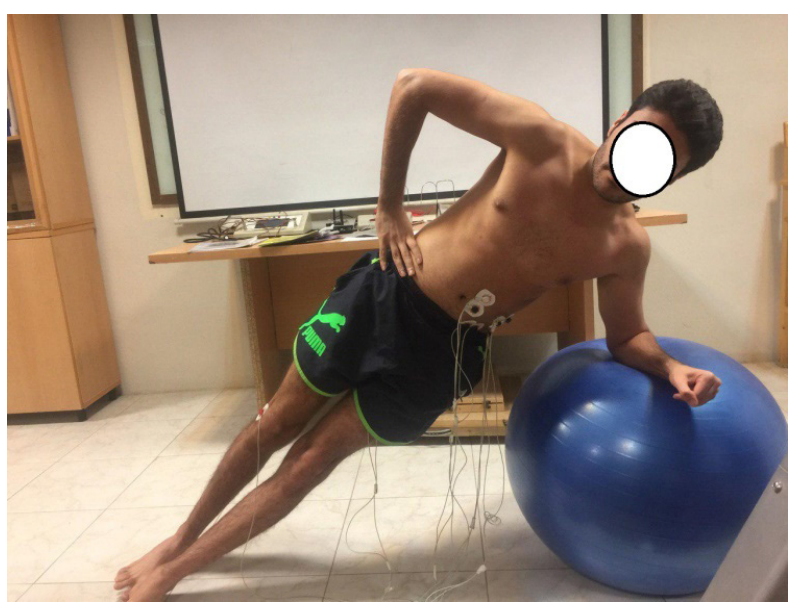

تصوير ا. حركت يلانك با و بدون توب سوئيسبال 
جدول ז. نتايج فعاليت الكترومايوكرافى عضلات موردمطالعه در حركت بِالايك باو بدون توب سوئيسبال بر اساس آزمون تى همبسته

\begin{tabular}{|c|c|c|c|c|c|}
\hline \multirow{2}{*}{$\mathbf{P}$} & \multirow{2}{*}{ تى } & \multicolumn{2}{|c|}{ ميانغين ثانحراف معيار } & \multirow{2}{*}{ df } & \multirow{2}{*}{ متغيير } \\
\hline & & با سوئيسبال & بدون سوئيسبال & & \\
\hline$+1+\cdot \Delta^{*}$ & $-r / r \cdot 1$ & $.10 \Delta \pm \cdot / 18$ & $\cdot 10+ \pm+/ 19$ & if & راست شكمى \\
\hline$\cdot 11 \cdot r$ & $-1 / M^{2 q}$ & $. / M \pm \pm .1 .8$ & $\cdot 1 \cdot \pm r r / \cdot A$ & if & مورب داخلى \\
\hline $.1 . . r^{*}$ & $-r / W$. & $.18 \mathrm{r} \pm . / 18$ & $\cdot 108 \pm . / 11$ & if & هورب خارجى \\
\hline $.1 . .1 *$ & -8/RV & $. / T V \pm \cdot / N V$ & $\cdot / M \mid \pm \cdot / 18$ & If & سريني ميائي \\
\hline
\end{tabular}

مجله بيومكانيك وزنش

سرينى ميانى در حركت هيلانك از يهلو با توب سوئيسبال نسبت

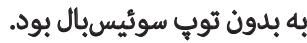

هريس و همكاران (Vا.IV) در تهقيقى كزارش كردند كه انجام

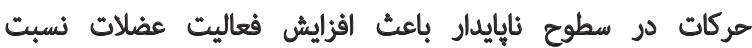

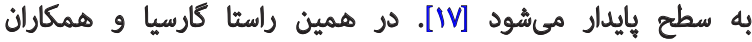

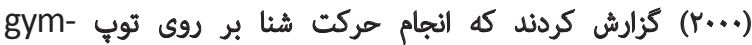

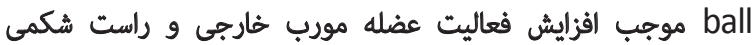

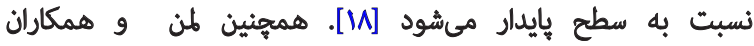

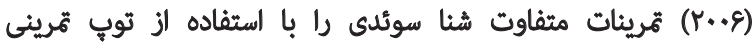

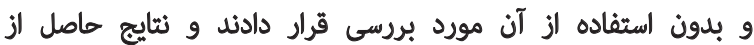

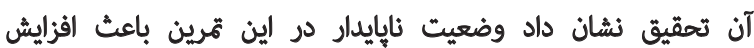

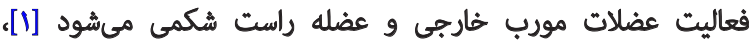

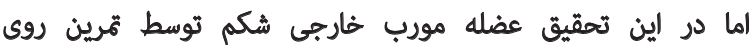

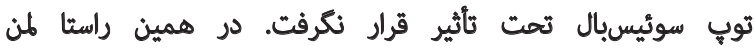

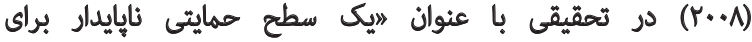

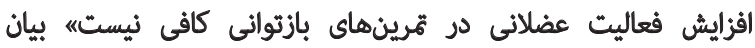

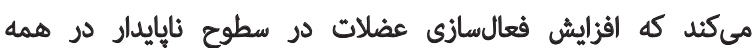

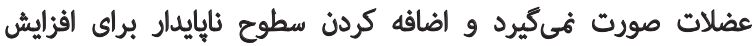

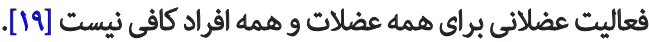
مارشال و همكاران (9.4) بيان كردند كه كاهش تعادل مربوط

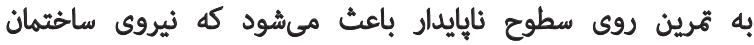

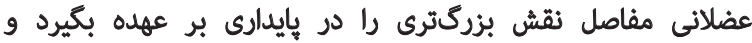

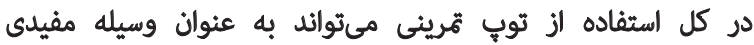

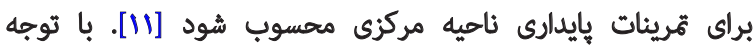

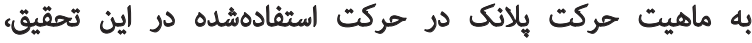

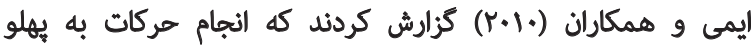

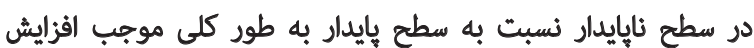

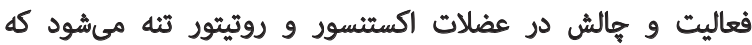
اين افزايش فعاليث با كنتزل اين حركت مرتبط است [ـr]]. در تهقيق حاضر استفاده از توبي سوئيسبال در اين ثمرين

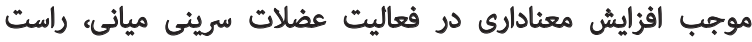
شكمى و مورب خارجى نسبت به سطع بايدار شد كه با نتايج
"اختلاف معنى الر در سطح آلفاى هـ • است.

و جرخش به راست تثنه وارد مىكند ["]].

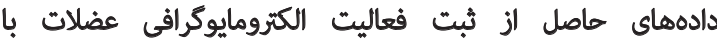

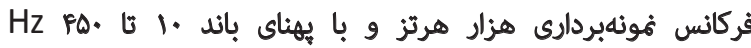

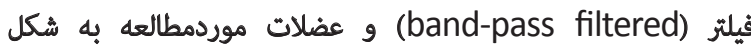
بارامتر RMS و به صورت درصدى از MVIC با كذاشتن در فرمول

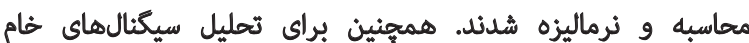

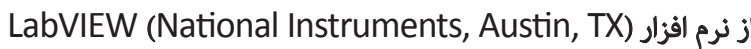

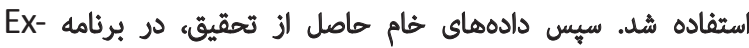

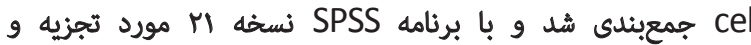

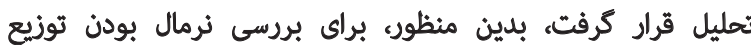

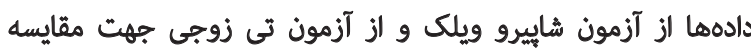

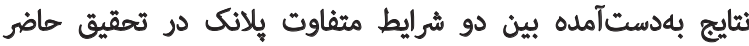

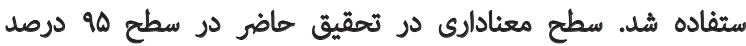

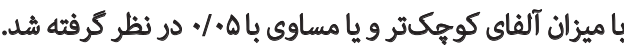

نتايج

دادههاى فعاليت الكترومايوكرافى الز عضلات موردمطالعه حين

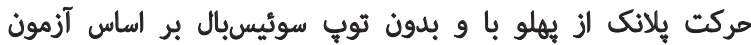

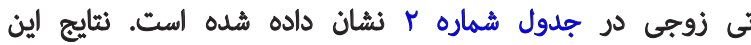

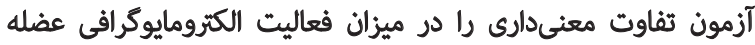

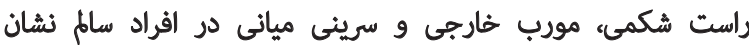

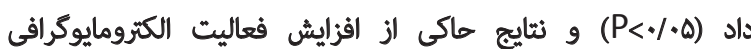

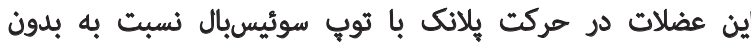

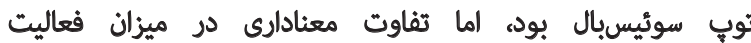

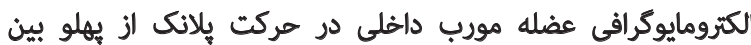

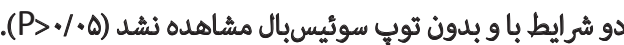

هدف مطالعه حاضر بررسى تأثير توب سوئيسبال بر فعاليت

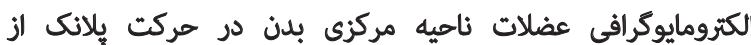

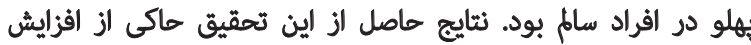

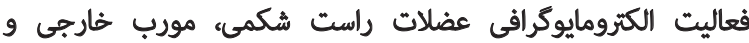




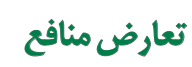

بنا به اظهار نويسندكان اين مقاله تعارض منافع ندارد.

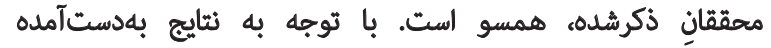

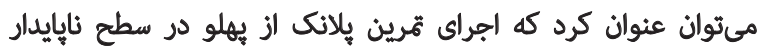

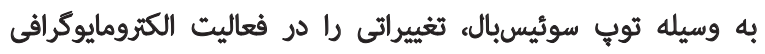

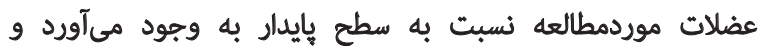

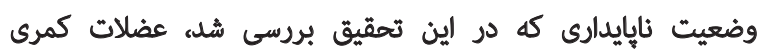

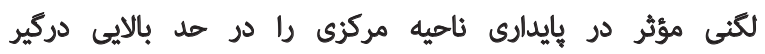

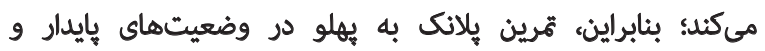

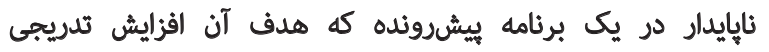

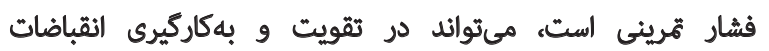
عضلات مؤثر در بايدارى ناحيه مركزى مؤثر باشد.

\section{نتيجلَّيرى نهايي}

در تهقيق حاضر، افزايش فعاليت عضلات سرينى ميانى، راست

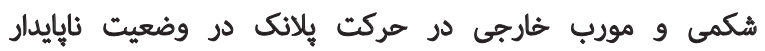

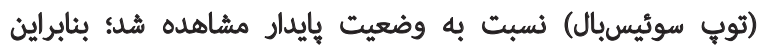

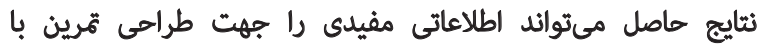

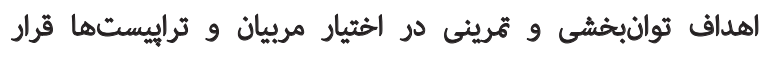

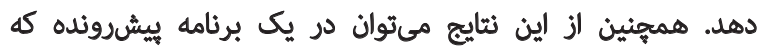

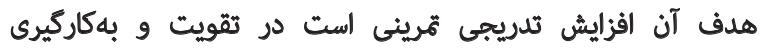

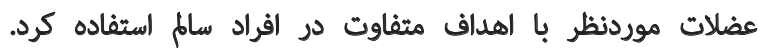

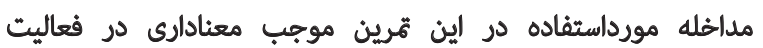

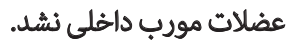

مطالعه حاضر با محدوديت هايى روبهرو بود. در مطالعه حاضر

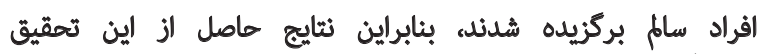

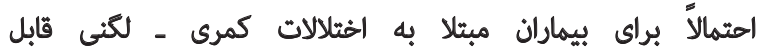

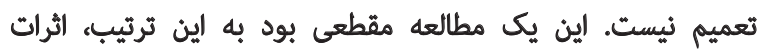

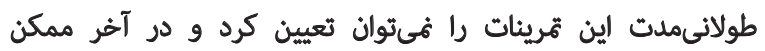

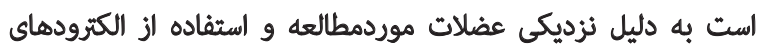
سطحى بين سيكنالهاي عضلات، Crosstalk اتفاق اقتاده باشد.

\section{مالاحظات اخلاقى}

$$
\text { بيروى از اصول اخلاق يُوهش }
$$

ثمامى آزمودنىها به طور داوطلبانه و با تكميل رضايتنامه

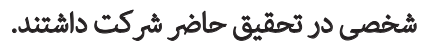

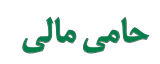

اين برؤش حاصل از طرح تحقيقاتى متعلق به دانشكاه علامه

$$
\text { طباطبايىاست. }
$$

$$
\text { مشاركت نويسند مكّان }
$$

مقام نويسندكان در آمادهسازى اين مقاله مشاركت داشتهاند. 


\section{References}

[1] Lehman GJ, Hoda W, Oliver S. Trunk muscle activity during bridging exercises on and off a swissball. Chiropractic \& osteopathy. 2005; 13:14. [DOI:10.1186/1746-1340-13-23] [PMID] [PMCID]

[2] Kim MJ, Oh DW, Park HJ. Integrating arm movement into bridge exercise: Effect on EMG activity of selected trunk muscles. Journal of Electromyography and Kinesiology. 2013; 23(5):1119-23. [DOI:10.1016/j. jelekin.2013.07.001] [PMID]

[3] Kim SY, Kang MH, Kim ER, Jung IG, Seo EY, Oh J. Comparison of EMG activity on abdominal muscles during plank exercise with unilateral and bilateral additional isometric hip adduction. Journal of Electromyography and Kinesiology. 2016; 30:9-14. [DOI:10.1016/j.jelekin.2016.05.003] [PMID]

[4] Snarr RL, Esco MR. Electromyographical comparison of plank variations performed with and without instability devices. ournal of Strength and Conditioning Research. 2014; 28(11):3298-305. [DOI:10.1519/ JSC.0000000000000521] [PMID]

[5] Cuğ M, Ak E, Özdemir RA, Korkusuz F, Behm DG. The effect of instability training on knee joint proprioception and core strength. Journal of Sports Science \& Medicine. 2012; 11(3):468-74. [PMID] [PMCID]

[6] Byrne JM, Bishop NS, Caines AM, Crane KA, Feaver AM, Pearcey GEP. Effect of using a suspension training system on muscle activation during the performance of a front plank exercise. Journal of Strength and Conditioning Research. 2014; 28(11):3049-55. [DOI:10.1519/ JSC.0000000000000510] [PMID]

[7] Santana JC, Vera-Garcia FJ, McGill SM. A kinetic and electromyographic comparison of the standing cable press and bench press. Journal of Strength and Conditioning Research. 2007; 21(4):1271. [DOI:10.1519/R-20476.1] [PMID]

[8] Goodman CA, Pearce AJ, Nicholes CJ, Gatt BM, Fairweather IH. No difference in $1 \mathrm{RM}$ strength and muscle activation during the barbell chest press on a stable and unstable surface. Journal of Strength and Conditioning Research. 2008; 22(1):88-94. [DOI:10.1519/JSC.0b013e31815ef6b3] [PMID]

[9] Fowles JR. What I always wanted to know about instability training. Applied Physiology, Nutrition, and Metabolism. 2010; 35(1):89-90. [DOI:10.1139/H09-134] [PMID]

[10] Behm DG, Leonard AM, Young WB, Bonsey WAC, MacKinnon SN. Trunk muscle electromyographic activity with unstable and unilateral exercises. Journal of Strength and Conditioning Research. 2005; 19(1):193-201. [DOI:10.1519/1533-4287(2005)192.0.CO;2] [PMID]

[11] Marshall P, Murphy B. Changes in muscle activity and perceived exertion during exercises performed on a swiss ball. Applied Physiology, Nutrition, and Metabolism. 2006; 31(4):376-83. [DOI:10.1139/h06-006] [PMID]

[12] Boling M, Padua D, Troy Blackburn J, Petschauer M, Hirth C. Hip adduction does not affect VMO EMG amplitude or VMO: VL ratios during a dynamic squat exercise. Journal of Sport Rehabilitation. 2006; 15(3):195205. [DOI:10.1123/jsr.15.3.195]

[13] Nathan AJ, Scobell A. How China sees America: The sum of Beijing's fears. Foreign Affairs. 2012; 91(5):32-47. https://www.rand.org/pubs/ external_publications/EP66518.html

[14] Slater LV, Hart JM. Muscle activation patterns during different squat techniques. The Journal of Strength \& Conditioning Research. 2017; 31(3):667-76. [DOI:10.1519/JSC.0000000000001323] [PMID]
[15] da Silva JJ, Schoenfeld BJ, Marchetti PN, Pecoraro SL, Greve JMD, Marchetti PH. Muscle activation differs between partial and full back squat exercise with external load equated. The Journal of Strength \& Conditioning Research. 2017; 31(6):1688-93. [DOI:10.1519/ JSC.0000000000001713] [PMID]

[16] Lee JH, Cynn HS, Kwon OY, Yi CH, Yoon TL, Choi WJ, et al. Different hip rotations influence hip abductor muscles activity during isometric sidelying hip abduction in subjects with gluteus medius weakness. Journal of Electromyography and Kinesiology. 2014; 24(2):318-24. [DOI:10.1016/j. jelekin.2014.01.008] [PMID]

[17] Harris S, Ruffin E, Brewer W, Ortiz A. Muscle activation patterns during suspension training exercises. International Journal of Sports Physical Therapy. 2017; 12(1):42-52. [PMID] [PMCID]

[18] Vera-Garcia FJ, Grenier SG, McGill SM. Abdominal muscle response during curl-ups on both stable and labile surfaces. Physical Therapy. 2000; 80(6):564-9. [DOI:10.1093/ptj/80.6.564] [PMID]

[19] Lehman GJ, Gilas D, Patel U. An unstable support surface does not increase scapulothoracic stabilizing muscle activity during push up and push up plus exercises. Manual Therapy. 2008; 13(6):500-6. [DOl:10.1016/j.math.2007.05.016] [PMID]

[20] Imai A, Kaneoka K, Okubo Y, Shiina I, Tatsumura M, Izumi S, et al. Trunk muscle activity during lumbar stabilization exercises on both a stable and unstable surface. Journal of Orthopaedic \& Sports Physical Therapy. 2010; 40(6):369-75. [DOI:10.2519/jospt.2010.3211] [PMID] 
This Page Intentionally Left Blank 